\title{
Perilesional Linear Atrophic Streaks Associated with Intralesional Corticosteroid Injections in a Psoriatic Plaque
}

\author{
Aditya K. Gupta, M.D.* and James E. Rasmussen, M.D.* $\uparrow$ \\ Departments of ${ }^{*}$ Dermatology and ${ }^{*}+$ Pediatrics, University of Michigan Medical Center, \\ Ann Arbor, Michigan.
}

\begin{abstract}
Perilymphatic atrophy can be a complication of intralesional corticosteroid injections given for the treatment of conditions such as psoriasis, alopecia areata, and keloids, and intraarticular corticosteroid injections given in diseases such as rheumatoid arthritis. It may become manifest as perilesional linear, depigmented, atrophic streaks, which are usually most prominent in patients with dark-colored skin.
\end{abstract}

Numerous cutaneous changes have been reported after systemic and local corticosteroid administration $(1,2)$, for example, local atrophy of the skin and subcutaneous tissue. Linear atrophic streaks, presumed to be atrophy of the dermis overlying lymphatic vessels draining the injection site, were reported after intralesional corticosteroid injections into a lesion of alopecia areata $(3,4)$ and keloid $(4,5)$, and intraarticular injections into knees (6). This report may promote more frequent recognition of this side effect, which should be kept in mind when intralesional corticosteroid injections are given in psoriasis and other diseases.

\section{CASE REPORT}

A 15-year-old black girl was first seen by us in June 1986 for the evaluation of a lesion on the lateral aspect of her left foot that had been present for the last several months. Previous treatments included three intralesional corticosteroid injections (triamcinolone, $2.5 \mathrm{mg} / \mathrm{ml}$ ) as well as topical corticosteroids, resulting in a moderate response.

On examination, on the lateral aspect of her left foot was a well-demarcated, erythematous, scaly plaque, approximately $4 \times 1.5 \mathrm{~cm}$ in diameter (Fig. 1). No other cutaneous lesions were present elsewhere. Clinically, the diagnosis was psoriasis. From the anterosuperior edge of the plaque, an inverted Y-shaped streak of atrophy with depigmentation was noted. A vertical streak was also present over posterosuperior aspect of the plaque (Fig. 1). The former was $8 \mathrm{~mm}$ wide and the combined length of the three links of the Y-shaped streak was approximately $9 \mathrm{~cm}$. The latter was $1.2 \mathrm{~cm}$ wide and $4 \mathrm{~cm}$ long.

The patient was otherwise in good health and was taking no medication. There was no family history of psoriasis or other cutaneous disease. When seen in follow-up six weeks later, the perilesional streaks were almost completely resolved. 


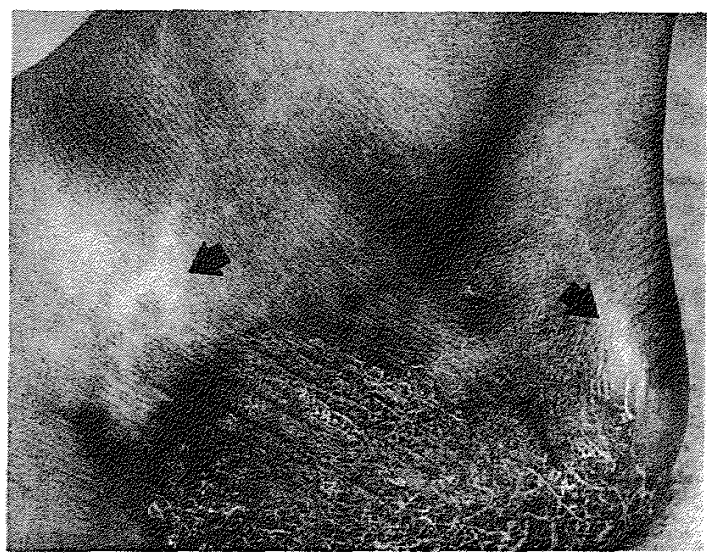

Figure 1. Psoriatic plaque on the lateral aspect of the left foot. Along the anterosuperior and posterosuperior borders there are streaks of atrophy and depigmentation.

\section{COMMENT}

Two pronounced, depressed streaks developed in a patient with alopecia areata after two or three intralesional injections of corticosteroids (3). It was assumed that the corticosteroid was transmitted by the lymphatic vessels.

Linear atrophy was described extending distally on the forehead starting from a lesion of alopecia areata into which intradermal corticosteroids had been injected (4). Upon injection of local anesthetic prior to obtaining a biopsy specimen, the atrophic line disappeared. When Evan blue was injected along the atrophic line, it was found that a lymphatic vessel lay in the same path.

Linear atrophy was reported after intralesional injections of triamcinolone in a keloid located on an arm (5). A Y-shaped lesion with a total length of 17 $\mathrm{cm}$ developed, starting at the proximal and distal aspects of the keloid. Alphazurine $2 G$ (patent blue) was injected at two points along the atrophic line using the same technique as that employed to find a lymphatic vessel during lymphangiography. The atrophic line ran along the course of the vessel, which stained blue at both points. Histologic examination showed the stained vessel to be atonic and partly lined by cuboidal cells. These points were in favor of the vessel being lymphatic in origin.

Linear skin atrophy was associated with repeated intraarticular corticosteroid injections into the knees of a patient with severe rheumatoid ar- thritis (6). During a three-year period, approximately 40 injections of triamcinolone acetonide, triamcinolone hexacetonide, dexamethasone acetate. or methylprednisolone were administered to both knees at various times. Generally, an intraarticular injection of 20 to $40 \mathrm{mg}$ of a triamcinolone compound (or an equivalent amount of another corticosteroid) was given at any one time.

The atrophic perilesional streaks seen in our patient were associated with intralesional corticosteroid injections given to treat the plaque of psoriasis, and resembled those appearing after the same form of therapy as cited. Our patient declined to have a biopsy or lymphangiogram. We speculate that the perilesional atrophic streaks in our patient represented perilymphatic atrophy, as in the other cases.

Fine suspension of corticosteroids is probably removed by the perilesional lymphatics. If a high enough intravascular corticosteroid concentration is reached, this may predispose to perilymphatic atrophy. This likely depends upon the number and frequency of the intralesional injections and the potency of corticosteroid used. Abnormal permeability of perilesional lymphatics, present in association with active disease in the lesion, could be a predisposing factor.

Perilymphatic atrophy may be permanent, but gradual resolution has been reported (3-5). In our patient, when seen in follow-up six weeks later, the perilesional atrophic streaks had almost disappeared.

This report should raise the index of suspicion for perilymphatic atrophy when perilesional linear atrophic streaks are noted after intralesional or intraarticular injections of corticosteroids. These may be more prominent in patients who have a dark complexion.

\section{REFERENCES}

1. Cook TJ, Lorincz AL. Absence of steroid acne in children. Arch Dermatol 1964;89:442-445.

2. Hurley HJ. Dermatology. In: Moschella SL, Pillsbury DM, Hurley HI, eds. Philadelphia: WB Saunders, 1975:1626.

3. Ayres S Jr. Alopecia areata with associated subcutaneous atrophy. Arch Dermatol 1964;90:242.

4. Kikuchi 1, Horikawa S. Perilymphatic atrophy of the skin. Arch Dermatol 1975;111:795-796.

5. Kikuchi I, Horikawa S. Perilymphatic atrophy of the skin. Arch Dermatol 1974;109:558-559.

6. Gottlieb NL, Penneys NS, Brown HE Jr. Periarticular perilymphatic skin atrophy. JAMA 1978;240:559-560. 
This document is a scanned copy of a printed document. No warranty is given about the accuracy of the copy. Users should refer to the original published version of the material. 